\title{
ANÁLISIS DE LA INTERACCIÓN VERBAL ENTRE PROFESORES Y ESTUDIANTES DE ENFERMERÍA EN CONTEXTOS DE AULA Y HOSPITAL. UN ESTUDIO DESCRIPTIVO
}

\author{
Analysis of verbal interaction between teachers and \\ students of nursing in classroom and hospital contexts. \\ A descriptive study
}

\section{L'analyse de l'interaction verbale entre les enseignants et les étudiants de soins infirmiers dans les contextes de classe et de l’hôpital. Une étude descriptive}

María Josefa Benavente SANGuino*, Manuel LuCERO Fustes*** y Sixto CuBo Delgado**** * Escuela de Enfermería de la Comunidad (Consejería de Salud y Politica Social) adscrita a la Universidad de Extremadura

** Facultad de Educación. Departamento de Ciencias de la Educación. Área de Didáctica y Organización Escolar. Universidad de Extremadura * Facultad de Educación. Departamento de Ciencias de la Educación. Área de Métodos de Investigación y Diagnóstico en Educación. Universidad de Extremadura.Correo-e: mlucero@unex.es

Recibido: 30-01-2013; Aceptado: 18-10-2013; Publicado: 30-03-2014 BIBLID [0212-5374 (2014) 32, 1; 117-139]

Ref. Bibl. MARÍA JOSEFA BENAVENTE SANGUINO, MANUEL LUCERO FUSTES y SIXTO CUBO DELGADO. Análisis de la interacción verbal entre profesores y estudiantes de Enfermería en contextos de aula y hospital. Un estudio descriptivo. Enseñanza \& Teaching, 32, 1-2014, 117-139. 
MARÍA JOSEFA BENAVENTE SANGUINO, MANUEL LUCERO FUSTES Y SIXTO CUBO DELGADO ANÁLISIS DE LA INTERACCIÓN VERBAL ENTRE PROFESORES Y ESTUDIANTES DE ENFERMERÍA EN CONTEXTOS DE AULA Y HOSPITAL. UN ESTUDIO DESCRIPTIVO

RESUMEN: El objetivo fundamental es identificar, describir y documentar el discurso que se produce en la enseñanza y el aprendizaje de la Enfermería. Desde el punto de vista metodológico, se adopta una estrategia observacional de estudio de dos casos y se propone un modelo de análisis formado por diversas unidades y niveles. Más concretamente, se describen y comparan las explicaciones que realizan los profesores del Grado de Enfermería en torno a dos procedimientos clínicos: la toma de presión arterial y la punción venosa, en dos contextos diferentes de enseñanza como son las unidades de enfermería de hospitales y las escuelas de enfermería. Para ello, se han examinado todas las interacciones en torno a las subtareas de cada procedimiento; se ha indagado en las subtareas que conforman el antes, durante y después de cada práctica; se han comparado las participaciones con diferente grado de dificultad, y se ha intentado profundizar en el proceso de traspaso de control de los estudiantes. Los resultados muestran tres importantes diferencias en las explicaciones de los profesores en función del contexto (universidad u hospital): a) el profesorado de la Escuela de Enfermería otorga más importancia al aprendizaje de los puntos críticos establecidos para cada procedimiento; b) los aspectos técnicos priman en la Escuela de Enfermería y los referidos al confort y bienestar del paciente en el Hospital; c) en la Escuela de Enfermería apenas se constataron episodios que mostraran una interacción más o menos simétrica o con un grado de participación alto de los estudiantes. En las unidades de enfermería, por el contrario, se incrementaba el nivel de responsabilidad y la autonomía de los alumnos.

Palabras clave: enseñanza universitaria, toma de la presión arterial, punción venosa, actividad conjunta, ayuda educativa, discurso educacional, interacción educativa, prácticas educativas.

SUMMARY: The fundamental objective is to identify, describe and document the discourse produced in the teaching and learning of nursing. From a methodological viewpoint, an observational strategy of case study of two cases is adopted and a method of analysis based on several units and levels is proposed. Specifically, the explanations which teachers of the Degree in Nursing give regarding two clinical procedures are described and compared: taking blood pressure and venipuncture; in two different contexts of teaching: nursing units in hospitals and nursing schools. In order to do so, all the interactions surrounding the tasks in each procedure have been examined; the subtasks in the pre-, while- and post-practice activities have been explored; the participation with different degrees of difficulty have been compared and an attempt has been made to elucidate the process of control transfer to the students. The results show significant differences in the explanations of teachers depending on the context (university or hospital): a) the teachers at the nursing school give more importance to the learning of critical points established for each procedure, b) technical aspects take precedence in the nursing school whereas those regarding the comfort and well being of the patient do so in the hospital, c) in the nursing school hardly any episodes were found that showed a more or less symmetrical interaction or a high level of participation of students. On the contrary, in the nursing units, the level of responsibility and autonomy of the students increased. 
MARÍA JOSEFA BENAVENTE SANGUINO, MANUEL LUCERO FUSTES Y SIXTO CUBO DELGADO ANÁLISIS DE LA INTERACCIÓN VERBAL ENTRE PROFESORES Y ESTUDIANTES DE ENFERMERÍA EN CONTEXTOS DE AULA Y HOSPITAL. UN ESTUDIO DESCRIPTIVO

Key words: higher education, taking blood pressure, venipuncture, joint activity, educational support, educational discourse, educational interaction, educational practices.

RÉSUMÉ: L'objectif fondamental est d'identifier, de décrire et de documenter le discours qui se produit dans l'enseignement et l'apprentissage des soins infirmiers. Du point de vue méthodologique, on adopte une stratégie observationnelle d'étude de deux cas et on propose un modèle d'analyse qui est formé par diverses unités et niveaux. Plus précisément, sont décrites et comparées les explications qui effectuent les professeurs de Licence en Soins Infirmiers sur deux procédures cliniques: prenant la tension artérielle et ponction veineuse, dans deux contextes d'enseignement différents, tels que les unités de soins des hôpitaux et des écoles de soins infirmiers. Pour ce faire, ont été examinés toutes les interactions environ les sous-tâches de chaque procédure; ont été étudiées les sous-tâches comprenant l'avant, pendant et après de chaque pratique; ont été comparés les actions avec différents degrés de difficulté, et a tenté d'approfondir le processus de remise de contrôle a les élèves. Les résultats montrent trois différences importantes dans les explications des enseignants dans des contextes différents (université ou hôpital): a) le corps professoral de l'École des sciences infirmières donne plus d'importance à l'apprentissage des points critiques établis pour chaque procédure; b) les aspects techniques ont plus prévalent à l'École des sciences infirmières et les associés au confort et bien-être du patient à l'hôpital; c) dans l'École des sciences infirmières à peine ont été constatées épisodes avec une interaction symétrique plus ou moins ou avec un haut degré de participation des étudiants. Dans les unités de soins, au contraire, il a augmenté le niveau de responsabilité et d'autonomie des étudiants.

Mots clés: l'enseignement universitaire, prendre la tension artérielle, ponction veineuse, activité conjointe, aide à l'éducation, discours éducatif, interaction éducative, pratiques éducatives.

\section{INTRODUCCIÓN}

En el ámbito de la salud y, más concretamente, en lo que se refiere a la formación de profesionales de la Enfermería, existe una gran preocupación por determinar cómo han de ser educados los estudiantes para que lleguen a ser unos profesionales competentes en las condiciones particulares en las que se ejerce esta profesión. La razón fundamental que ha motivado la realización de este estudio radica no solo en esta preocupación, sino también en la escasez de trabajos que aborden la relación entre la teoría enfermera y su aplicación en la práctica. Es una fuente de desasosiego para una buena parte del colectivo profesional que frecuentemente se cuestiona el alejamiento entre el discurso teórico de la Enfermería y la práctica cuidadora. Esta inquietud por las discrepancias entre la teoría y la práctica es perceptible desde hace años y ha sido expresada formalmente por diversos organismos internacionales en documentos muy relevantes (Comité Consultivo para la Formación de Enfermeros de la Comisión de 
MARÍA JOSEFA BENAVENTE SANGUINO, MANUEL LUCERO FUSTES Y SIXTO CUBO DELGADO ANÁLISIS DE LA INTERACCIÓN VERBAL ENTRE PROFESORES Y ESTUDIANTES DE ENFERMERÍA EN CONTEXTOS DE AULA Y HOSPITAL. UN ESTUDIO DESCRIPTIVO

las Comunidades Europeas, 1990; Consejo General de Enfermería de España, 1992; Consejo Internacional de Enfermeras, 2008).

\section{MARCO TEÓRICO}

\subsection{Contexto actual de la enseñanza y el aprendizaje de la enfermería}

Tradicionalmente las relaciones entre el conocimiento teórico y la práctica educativa ha estado marcada por un planteamiento positivista en el que muchos docentes de los distintos niveles educativos señalan que existe una clara desconexión entre el conocimiento teórico y la práctica educativa real (Coll, 2010; Cubero, 2010).

La cuestión de la relación teoría-práctica adquirió gran interés, sobre todo después de los trabajos de Schön (1992) y Benner (1987), que marcaron un punto de inflexión en la comprensión de esa relación, tanto en la formación de profesores como en la de enfermeras. Estos trabajos reestimularon los debates acerca de la naturaleza de las nociones de teoría y práctica, y de su relación, siendo desde entonces una de las principales preocupaciones de la literatura en los ambientes académicos de la Enfermería. Trabajos como los de Ashworth y Longmate (1993) o Connant (1992) señalan la existencia de una dicotomía y/o vacío y su necesaria superación.

Posteriormente, en un estudio de Carnwell, Baker, Bellis y Murray (2007), se exploran las diferencias entre tutores, profesores, tutores de enlace y cómo funcionan en conjunto para ayudar a los estudiantes a integrar la teoría y la práctica. El análisis de contenido cualitativo del estudio puso sobre la mesa varios puntos de divergencias: las distintas competencias, las diferencias del rol y los conflictos de roles. Sharples, Kelly y Elcock (2007) describieron los esfuerzos de la Thames Valley University por prestar apoyo a los tutores en la práctica clínica mediante la introducción de asesores educativos en todas las unidades de aprendizaje. Myall, Levett-Jones y Lathlean (2008) exploraron el papel del tutor de prácticas en la actual enseñanza de Enfermería en el Reino Unido utilizando estudios precedentes realizados en Australia sobre las percepciones de los estudiantes de Enfermería y los tutores. Más recientemente Andrew y Robb (2011) siguen cuestionando el papel académico (al que consideran controvertido) y la credibilidad clínica de la Enfermería en el Reino Unido. Por otro lado, Jokelainen, Turunen, Tossavainen, Jamookeeah, y Coco (2011) analizaron, mediante un análisis de contenido, los artículos de investigación publicados en un periodo de 20 años (1986-2006). Establecieron que no hay acuerdo sobre asesoramiento a los estudiantes, pues los enfoques de la tutoración varían. Concluyeron que los beneficios del establecimiento de normas y requerimientos básicos para las tutorías y el asesoramiento de los estudiantes deben ser revisados sistemáticamente para reflejar los cambios que se producen en la Enfermería y en la educación, de forma que se mantenga actualizada la práctica. Según las autoras, unificar el asesoramiento de los estudiantes, aseguraría la calidad en el aprendizaje y facilitaría la contratación de los futuros profesionales. 
MARÍA JOSEFA BENAVENTE SANGUINO, MANUEL LUCERO FUSTES Y SIXTO CUBO DELGADO

ANÁLISIS DE LA INTERACCIÓN VERBAL ENTRE PROFESORES Y ESTUDIANTES DE ENFERMERÍA EN CONTEXTOS DE AULA Y HOSPITAL. UN ESTUDIO DESCRIPTIVO

\subsection{La interacción en la enseñanza}

La investigación reciente ha considerado el aula como un lugar en el que se producen complejas interacciones y donde se transmite el conocimiento. Así entendida, la interacción didáctica es un componente que ha de construir y mejorar continuamente el profesor. Durante los últimos años ha cobrado un creciente interés el estudio de la vida en las aulas, con objeto de observar y documentar las interacciones que se producen entre los profesores y alumnos. En este sentido, una de las líneas de investigación que han estudiado las formas de recoger y analizar lo que hacen y dicen los profesores durante la interacción en las aulas es aquella en la que se concibe la enseñanza como un proceso comunicativo en el que los interlocutores participan para la construcción de comprensiones conjuntas. Autores como Edwards y Mercer (1988) y Mercer (2001) centran sus trabajos en describir las regularidades y rasgos típicos de la vida en las aulas así como la utilización del lenguaje por parte de los profesores y alumnos de cara a la construcción del conocimiento (Sánchez y Rosales, 2005; García, Rosales y Sánchez, 2003; Sánchez, García, Rosales, De Sixte y Castellano, 2008). El sistema de análisis se basa en la grabación, entrevista a los profesores y análisis de las transcripciones con el fin de esclarecer las regularidades presentes en el discurso de los profesores y sus alumnos.

Otros trabajos se centran más bien en analizar la evolución que experimentan las actividades desarrolladas en una unidad didáctica, prestando una especial atención al proceso de transferencia del control de la responsabilidad en la ejecución de una tarea determinada. De acuerdo con esto, el análisis de la interactividad se centra en la articulación de las actuaciones de profesor y alumnos en torno a un determinado contenido o tarea específica de aprendizaje (Coll, Colomina, Onrubia y Rochera, 1995).

Fruto de estos modelos de análisis, algunos resultados de investigaciones realizadas sobre las explicaciones de profesores de educación primaria y secundaria ponen de manifiesto que, en las actividades de carácter expositivo, los profesores asumen casi toda la responsabilidad en la elaboración de las ideas, imponiendo estructuras muy restrictivas a los roles y acciones de los alumnos. De los alumnos se espera que tomen notas, que transcriban o reelaboren esa información y que, puntualmente, hagan preguntas sobre aquello que no entienden. En cambio, en los episodios de aplicación o supervisión del aprendizaje, la participación del alumno se hace a menudo más explícita a través de su respuesta a las preguntas o problemas que le presenta el profesor. El control de la participación que ejerce este tipo de preguntas e indicaciones suele variar desde una dirección muy estrecha de la participación del alumno hasta una cierta transferencia de la responsabilidad en la actividad conjunta sobre la tarea (Montanero y García, 2005). En esta misma línea, un estudio observacional de caso múltiple que pretendía documentar las explicaciones de varios profesores de Secundaria en torno a un determinado fenómeno histórico puso de manifiesto las grandes dificultades de los profesores 
MARÍA JOSEFA BENAVENTE SANGUINO, MANUEL LUCERO FUSTES Y SIXTO CUBO DELGADO ANÁLISIS DE LA INTERACCIÓN VERBAL ENTRE PROFESORES Y ESTUDIANTES DE ENFERMERÍA EN CONTEXTOS DE AULA Y HOSPITAL. UN ESTUDIO DESCRIPTIVO

para ayudar a sus estudiantes a comprender e implicarse en la comprensión de las relaciones causales entre determinados fenómenos históricos (Lucero y Montanero, 2008; Montanero y Lucero, 2011).

En el ámbito universitario, otros resultados han demostrado que las ayudas que los profesores ofrecen a los alumnos con pocos conocimientos previos y que muestran dificultades relevantes en la realización de las tareas son, mayoritariamente, ayudas muy explícitas dirigidas a resolver directamente la tarea como hacer demostraciones explícitas o pautarles muy detalladamente los pasos a realizar. En cambio, en el caso de alumnos con altos conocimientos previos, el profesor ofrece inicialmente ayudas dirigidas a pautar de manera muy general los pasos a realizar y confirmar puntualmente que lo que están haciendo es correcto (Cubero, Santamaría, De la Mata, Prados y Bascón, 2005; Cubero, Cubero, Santamaría, De la Mata Benítez, Ignacio y Prados, 2008).

Estudios más actuales se centran en describir cómo los estudiantes inician la interacción en las clases, y cómo los profesores guían esta participación. En este sentido, Shepher (2012) analizó las estrategias discursivas utilizadas por los alumnos de entre 8 y 9 años al iniciar la interacción y la evaluación que los profesores hacían de las mismas. Los resultados de este estudio muestran que los estudiantes regularmente inician interacciones dentro de la clase, pero en la mayoría de las ocasiones fuera de las secuencias IRE/IRF, y sin el permiso del profesor, lo que supone que estas intervenciones sea ignoradas o reprimidas.

También, en los últimos años, ha aumentado el número de estudios que están analizando la interacción profesor-alumnos en la formación a distancia. En este sentido, la interacción también es la clave para el logro de aprendizajes de calidad (García Aretio, 2003). En la mayoría de estos estudios, se analizan los temas de conversación en torno a los cuales se discute y los procesos de negociación y construcción conjunta de significados que los participantes mantienen (Onrubia, Naranjo y Segués, 2009; Coll, De Gispert y Rochera, 2010). Los resultados parecen indicar que la calidad de los productos obtenidos de la formación virtual, está determinada en gran parte por la naturaleza de dicha interacción (Cabero, 2004). En este sentido, el mero intercambio de mensajes no es un indicador fiable de la existencia de un diálogo significativo (Onrubia, Naranjo y Segués, 2009).

En nuestro trabajo pretendemos realizar un estudio exploratorio de una importante dimensión de la enseñanza y el aprendizaje de la Enfermería: la práctica profesional. Para ello se han documentado y comparado las explicaciones de los profesores de Enfermería en torno a dos procedimientos desarrollados en diferentes contextos: la Unidad de Enfermería de un Hospital y el Aula de Demostración de una Escuela de Enfermería. De forma más concreta, nuestro propósito es identificar, describir y documentar la adquisición de determinadas competencias profesionales propias de las ciencias de la salud, caracterizando con cierto detalle la responsabilidad en el aprendizaje asumida por el profesor y los alumnos. 
MARÍA JOSEFA BENAVENTE SANGUINO, MANUEL LUCERO FUSTES Y SIXTO CUBO DELGADO

ANÁLISIS DE LA INTERACCIÓN VERBAL ENTRE PROFESORES Y ESTUDIANTES DE ENFERMERÍA EN CONTEXTOS DE AULA Y HOSPITAL. UN ESTUDIO DESCRIPTIVO

3. MÉTODO

\subsection{Participantes}

En el estudio participaron dos profesores de Enfermería de la Escuela de la Comunidad (Consejería de Salud y Política Social), tres enfermeras asistenciales del Hospital Universitario Infanta Cristina y 16 estudiantes de Enfermería en prácticas. Los profesores intervinientes forman parte de la plantilla de la Escuela y fueron seleccionados intencionalmente por ser los encargados de impartir los procedimientos elegidos. Todos tienen más de diez años de experiencia clínica, más de 20 años de experiencia docente y aceptaron voluntariamente participar en el estudio.

Las enfermeras asistenciales fueron escogidas teniendo en consideración una experiencia profesional no menor de cinco años, la colaboración asidua en la formación de estudiantes de la Escuela de Enfermería de la Comunidad y la aceptación voluntaria de su participación.

TABLA 1

Distribución de los participantes

\begin{tabular}{|c|c|c|c|c|c|c|c|}
\hline CASO & LUGAR & $\begin{array}{l}\text { SEXO DEL } \\
\text { PROFESOR }\end{array}$ & $\begin{array}{l}\text { AÑOS DE } \\
\text { EJERCICIO }\end{array}$ & $\begin{array}{c}\text { TAREA } \\
\text { GRABADA }\end{array}$ & $\begin{array}{l}\text { DURACIÓN } \\
\text { DE LA } \\
\text { GRABACIÓN }\end{array}$ & $\begin{array}{l}\text { CURSO DEL } \\
\text { ESTUDIANTE }\end{array}$ & $\begin{array}{c}\text { N } .^{\circ} \text { DE } \\
\text { ESTUDIANTES }\end{array}$ \\
\hline 1 & S.D. & Mujer & 34 & $\begin{array}{l}\text { Punción } \\
\text { Venosa }\end{array}$ & $25,8 \mathrm{~min}$ & $\begin{array}{l}2 .{ }^{\circ} \text { de } \\
\text { DUE }\end{array}$ & 6 \\
\hline 2 & S.D. & Hombre & 34 & Toma P.A. & $47,06 \mathrm{~min}$ & $\begin{array}{l}\text { 1. } .^{\mathrm{de}} \\
\text { Grado }\end{array}$ & 7 \\
\hline 3 & U.E. & Mujer & 15 & $\begin{array}{c}\text { Punción } \\
\text { Venosa }\end{array}$ & $7,34 \mathrm{~min}$ & $\begin{array}{l}\text { 2. } . \text { de } \\
\text { DUE }\end{array}$ & 1 \\
\hline 4 & U.E. & Mujer & 29 & $\begin{array}{l}\text { Punción } \\
\text { Venosa }\end{array}$ & $16,03 \mathrm{~min}$ & $\begin{array}{l}\text { 2. } . \text { de } \\
\text { DUE }\end{array}$ & 1 \\
\hline 5 & U.E. & Mujer & 5 & Toma P.A. & $6,10 \mathrm{~min}$ & $\begin{array}{l}2 . .^{\circ} \mathrm{de} \\
\text { DUE }\end{array}$ & 1 \\
\hline
\end{tabular}

S.D.: sala de demostración; U.E.: unidad de enfermería; P.A: presión arterial; DUE: diplomado universitario en enfermería.

En cuanto a los estudiantes, participaron siete de primer Curso de Grado y nueve de segundo Curso de la Diplomatura en Enfermería. Aceptaron voluntariamente colaborar y ser grabados. Fueron seleccionados accidentalmente al ser los que en el momento de la realización de las grabaciones participaban en los grupos de aprendizaje práctico o realizaban su periodo de aprendizaje clínico en el Hospital. Su participación en la Sala de Demostración de la Escuela o en las Unidades Clínicas obedece a la programación prevista para las enseñanzas práctico-clínicas, 
MARÍA JOSEFA BENAVENTE SANGUINO, MANUEL LUCERO FUSTES Y SIXTO CUBO DELGADO ANÁLISIS DE LA INTERACCIÓN VERBAL ENTRE PROFESORES Y ESTUDIANTES DE ENFERMERÍA EN CONTEXTOS DE AULA Y HOSPITAL. UN ESTUDIO DESCRIPTIVO

por lo que el emparejamiento con los profesores de la Escuela o con las enfermeras de las Unidades fue igualmente accidental como se muestra en la Tabla 1.

En las sesiones grabadas en el Hospital participan también pacientes y familiares. En todos los casos fueron informados del estudio y de la implicación que su participación suponía en él. Se obtuvo el permiso escrito de todos ellos.

\subsection{Diseño}

El presente estudio se plantea, concretamente, desde un enfoque socioconstructivista y sociolingüístico de análisis del discurso. Se trata de una aproximación pragmática de análisis del lenguaje en uso, en el marco de actividades de aprendizaje de la práctica enfermera. A través de una descripción de las formas lingüísticas de estas situaciones de aprendizaje y sus regularidades, pretendemos entender mejor los procesos típicos que caracterizan la interacción entre los estudiantes y los profesionales de Enfermería del ámbito asistencial y del ámbito académico.

Desde esta perspectiva, el estudio se fundamentó en un diseño observacional de caso múltiple. El objetivo principal fue el de analizar algunas características de la interacción y el apoyo que los profesionales de Enfermería proporcionaban al estudiante en actividades asistenciales habituales. Para ello se eligieron dos procedimientos de entre los que figuran en el programa formativo de los estudiantes y que son frecuentemente realizados por las enfermeras asistenciales. La selección no fue azarosa, sino que se tuvo en cuenta que éstos no conllevasen problemas de incomodidad, dolor, falta de intimidad o pudieran afectar la dignidad de los pacientes.

Se determinó en virtud de estos condicionantes que los dos procedimientos fueran: 1) Toma de presión arterial: procedimiento que tiene como propósito medir y registrar con precisión la presión que ejerce la sangre al pasar por las paredes de las arterias; 2) Punción venosa: tiene por finalidad la extracción de una muestra de sangre con fines analíticos o bien la administración de alguna sustancia por vía intravenosa.

El modo en que se realizan los procedimientos, responde a lo establecido en el Manual de Procedimientos de Enfermería del Hospital Infanta Cristina (1991), vigente en este centro y en cuya elaboración participó el profesorado de la Escuela de Enfermería de la Comunidad. Es por tanto conocido por todos los implicados en el estudio.

\subsection{Materiales}

Para obtener los registros audiovisuales de la interacción que se establecía entre las enfermeras y los estudiantes, se utilizó una cámara de grabación en formato mini-DV con micrófono externo. Las grabaciones, tanto en la Sala de Demostración como en las Unidades del Hospital, fueron efectuadas por una profesora de la Escuela de Enfermería, quien situó la cámara en diferentes planos y lugares de las estancias donde se llevaron a cabo las sesiones. 
MARÍA JOSEFA BENAVENTE SANGUINO, MANUEL LUCERO FUSTES Y SIXTO CUBO DELGADO

ANÁLISIS DE LA INTERACCIÓN VERBAL ENTRE PROFESORES Y ESTUDIANTES DE ENFERMERÍA EN CONTEXTOS DE AULA Y HOSPITAL. UN ESTUDIO DESCRIPTIVO

\subsubsection{Proceso de selección y homogeneización de los procedimientos}

En esta fase se revisaron y homogeneizaron los dos procedimientos seleccionados. Para los fines de esta investigación, el equipo de profesores intervinientes acordó establecer 20 pasos para la ejecución de los procedimientos seleccionados y agruparlos en tres momentos concretos de su desarrollo: antes, durante y después. El resultado de este trabajo de homogeneización, que respeta escrupulosamente las intervenciones recogidas en el Manual de Procedimientos, puede verse en las Tablas 2 y 3.

TABLA 2

Subtareas del Procedimiento de Toma de la Presión arterial. Las subtareas decisivas y más complicadas aparecen señaladas en las tablas con un asterisco (*)

\begin{tabular}{|c|c|}
\hline \multirow{7}{*}{ 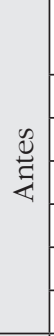 } & $\begin{array}{l}\text { 1. Definir el concepto y el propósito del procedimiento y la fundamentación de las } \\
\text { acciones. }\end{array}$ \\
\hline & 2. Seleccionar el material adecuado. \\
\hline & 3. Comprobar las órdenes. \\
\hline & 4. Lavarse las manos. \\
\hline & 5. Identificar al paciente. \\
\hline & 6. Valorar su estado. \\
\hline & 7. Informarle y tranquilizarle. \\
\hline \multirow{10}{*}{$\begin{array}{l}\stackrel{\mathscr{\Xi}}{\Xi} \\
\stackrel{\Xi}{\Xi} \\
\stackrel{\Xi}{\Xi}\end{array}$} & 8. Situar al paciente en una posición adecuada (sentado o acostado). \\
\hline & $\begin{array}{l}\text { 9. Dejar libre la extremidad y colocar el brazo en apoyo, con la palma de la mano } \\
\text { hacia arriba. }\end{array}$ \\
\hline & 10. Palpar y detectar el pulso braquial $(*)$ \\
\hline & 11. Vaciar el aire del manguito antes de colocarlo. \\
\hline & $\begin{array}{l}\text { 12. Colocar el manguito correctamente: centrado encima de la arteria, } 2,5 \mathrm{~cm} \text { por } \\
\text { encima del pulso detectado. }\end{array}$ \\
\hline & $\begin{array}{l}\text { 13. Colocarse el fonendoscopio antes de insuflar el aire en el manguito y comprobar } \\
\text { que los auriculares están abiertos. }\end{array}$ \\
\hline & $\begin{array}{l}\text { 14. Localizar nuevamente el latido arterial y colocar sobre él la campana del } \\
\text { fonendoscopio. }\end{array}$ \\
\hline & $\begin{array}{l}\text { 15. Insuflar el aire en el manguito sin quitar los dedos de la arteria, hasta } 30 \mathrm{~mm} \text { de } \\
\mathrm{Hg} \text { por encima del último latido palpado. }\end{array}$ \\
\hline & $\begin{array}{l}\text { 16. Liberar la presión del manguito a una velocidad de } 2-3 \mathrm{~mm} \mathrm{de} \mathrm{Hg} / \mathrm{s} \text { para escuchar } \\
\text { los latidos que marcan la presión arterial (*). }\end{array}$ \\
\hline & $\begin{array}{l}\text { 17. Abrir completamente la válvula después de oír el último latido y retirar el manguito } \\
\text { de la extremidad. }\end{array}$ \\
\hline \multirow{3}{*}{$\begin{array}{l}\stackrel{\mathscr{U}}{\Xi} \\
\stackrel{0}{\mathscr{n}} \\
\stackrel{0}{0}\end{array}$} & 18. Dejar al paciente en posición cómoda y con el brazo cubierto. \\
\hline & 19. Comentar con el paciente las cifras de presión arterial, si es necesario. \\
\hline & 20. Guardar el equipo en su lugar y registrar los valores en la gráfica del paciente. \\
\hline
\end{tabular}


126 MARÍA JOSEFA BENAVENTE SANGUINO, MANUEL LUCERO FUSTES Y SIXTO CUBO DELGADO ANÁLISIS DE LA INTERACCIÓN VERBAL ENTRE PROFESORES Y ESTUDIANTES DE ENFERMERÍA EN CONTEXTOS DE AULA Y HOSPITAL. UN ESTUDIO DESCRIPTIVO

Durante las sesiones llevadas a cabo por el profesorado implicado, el equipo determinó que algunos de los pasos de los procedimientos constituían puntos críticos que determinaban el éxito de su ejecución. Puede definirse el punto crítico como el momento decisivo, dificultoso o complicado dentro del desarrollo de un procedimiento. Los puntos críticos se utilizan en la Escuela como criterios absolutos para la evaluación criterial. Se encontraron dos puntos críticos en cada procedimiento, que aparecen señalados en las Tablas 2 y 3 con un asterisco (*).

TABLA 3

Subtareas del Procedimiento de Punción Venosa. Las subtareas decisivas y más complicadas aparecen señaladas en las tablas con un asterisco (*)

\begin{tabular}{|c|c|}
\hline \multirow{7}{*}{$\frac{\mathscr{d}}{\stackrel{2}{Z}}$} & $\begin{array}{l}\text { 1. Definir el concepto y el propósito del procedimiento y la fundamentación de las } \\
\text { acciones. }\end{array}$ \\
\hline & 2. Seleccionar el material adecuado. \\
\hline & 3. Comprobar las órdenes. \\
\hline & 4. Lavarse las manos. \\
\hline & 5. Identificar al paciente. \\
\hline & 6. Valorar su estado. \\
\hline & 7. Informarle y tranquilizarle. \\
\hline \multirow{10}{*}{ 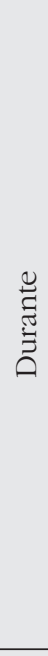 } & $\begin{array}{l}\text { 8. Colocar el compresor para valorar las venas antes de realizar la técnica. Luego se } \\
\text { retira (*). }\end{array}$ \\
\hline & 9. Desinfectar la zona correctamente y no contaminarla. \\
\hline & $\begin{array}{l}\text { 10. Colocar el compresor entre } 5 \text { y } 15 \mathrm{~cm} \text { por encima de la zona elegida para realizar } \\
\text { la extracción. }\end{array}$ \\
\hline & 11. Colocarse guantes de protección. \\
\hline & $\begin{array}{l}\text { 12. No tocar la zona elegida para la extracción sin desinfectarse previamente los } \\
\text { dedos. }\end{array}$ \\
\hline & $\begin{array}{l}\text { 13. Fijar la piel de la zona elegida para la extracción con la mano no dominante y con } \\
\text { la otra colocar la aguja con el bisel hacia arriba. }\end{array}$ \\
\hline & 14. Insertar la aguja en la vena seleccionada, con un ángulo de 30 a $45^{\circ}$ (*). $^{*}$ \\
\hline & $\begin{array}{l}\text { 15. Aspirar para comprobar que refluye la sangre y asegurar el punto de inserción con } \\
\text { un adhesivo. }\end{array}$ \\
\hline & $\begin{array}{l}\text { 16. Extraer la cantidad de sangre indicada para la prueba requerida y quitar el } \\
\text { compresor antes de retirar la aguja. }\end{array}$ \\
\hline & 17. Comprimir con una gasa seca la zona de punción y fijarlo con un esparadrapo. \\
\hline \multirow{3}{*}{$\frac{\mathscr{U}}{\stackrel{\mathscr{D}}{\mathscr{U}}}$} & $\begin{array}{l}\text { 18. Desechar el material contaminado en el biocontenedor de residuos, sin encapuchar } \\
\text { la aguja. }\end{array}$ \\
\hline & 19. Interesarse por el estado del paciente y dejarle cómodamente instalado. \\
\hline & \\
\hline
\end{tabular}


MARÍA JOSEFA BENAVENTE SANGUINO, MANUEL LUCERO FUSTES Y SIXTO CUBO DELGADO

ANÁLISIS DE LA INTERACCIÓN VERBAL ENTRE PROFESORES Y ESTUDIANTES DE ENFERMERÍA EN CONTEXTOS DE AULA Y HOSPITAL. UN ESTUDIO DESCRIPTIVO

\subsubsection{Procedimiento de análisis de la interacción}

El estudio de la interacción verbal se fundamentó en el análisis de los registros audiovisuales que se obtuvieron con cámara de vídeo en cada una de las sesiones grabadas y que fueron posteriormente trascritos en papel. A continuación describimos el procedimiento de segmentación y categorización de dichos registros, con objeto de reducir y analizar los datos cualitativos obtenidos.

\subsubsection{Segmentación de las unidades de análisis}

La fuente teórica que se tuvo principalmente en cuenta para diseñar el sistema inicial de categorías parte de estudios anteriores (Sánchez, García, Rosales, De Sixte y Castellano, 2008; Montanero y García, 2005 y Becerra, Montanero y Lucero, 2012), donde se realiza el análisis de la interacción verbal en contextos de mediación educativa. Más concretamente, en estos estudios se abordan diversos niveles de análisis, desde una perspectiva deductiva e inductiva. Contemplan la segmentación de la interacción en unidades globales como la actividad o el episodio; además de otras más específicas, como el ciclo y el turno. Para cada una de estas unidades, es posible, además, analizar diferentes dimensiones: el contenido temático-semántico (el qué); la estructura de participación o los patrones de discurso prevalecientes (el cómo); o el grado de contribución o autonomía mostrado por los alumnos a la hora de generar el contenido público de la interacción (el quién). Cada una de estas dimensiones se analiza de forma independiente. Cada una aporta información diferente sobre la práctica educativa. Así, el discurso se utiliza para caracterizar las propiedades educativas de distintas interacciones. Utilizando este sistema los investigadores han conseguido documentar aspectos muy relevantes de la práctica educativa de profesores expertos y principiantes en diferentes ámbitos.

En nuestro caso, los procedimientos clínicos se segmentaron en subtareas (cada uno de los pasos del procedimiento) más pequeñas, que permitieran un análisis más pormenorizado de la interacción y que serían identificadas en los diálogos entre el profesor de la Escuela (PE) y el estudiante de Enfermería (EE) y la enfermera de prácticas (EP) y el estudiante de Enfermería (EE), constituyendo estos contextos (Escuela y Unidad de Enfermería) dos unidades de análisis diferenciadas.

Para analizar el contenido de cada una de las subtareas, se optó por aislar específicamente los contenidos lingüísticos publicados en cada una de las intervenciones. Este proceso de segmentación permite realizar un análisis que puede aportar información cualitativa y cuantitativa de diversa naturaleza. Así, por ejemplo, en el siguiente fragmento referido al procedimiento de toma de presión arterial, puede observarse como el profesor se está refiriendo a la subtarea número 10, denominada "Palpar y detectar el pulso braquial":

Profesor: [...] se infla el manguito a 20 o $30 \mathrm{~mm}$ por encima de donde hemos dejado de detectar el pulso, empezamos a liberar aire ¿Con qué velocidad empezamos a liberar aire? La velocidad recomendada es entre 2 y $3 \mathrm{~mm}$ de mercurio ¿¿uhm?... 
MARÍA JOSEFA BENAVENTE SANGUINO, MANUEL LUCERO FUSTES Y SIXTO CUBO DELGADO ANÁLISIS DE LA INTERACCIÓN VERBAL ENTRE PROFESORES Y ESTUDIANTES DE ENFERMERÍA EN CONTEXTOS DE AULA Y HOSPITAL. UN ESTUDIO DESCRIPTIVO

2-3 $\mathrm{mm}$ de, de mercurio por segundo y, y estamos atentos a escuchar un primer latido. El primer latido pues, en la situación en que se encuentre la aguja, esa es la sistólica y continuamos escuchando latidos. Hay un momento que desaparece. En el momento ese de desaparecer, esa es la presión diastólica ¿de acuerdo?

\subsubsection{Categorización de las unidades de análisis}

\section{Dimensión 1. Categorización de las subtareas según el grado de dificultad}

Para estudiar la ejecución de los procedimientos, era necesario delimitar las operaciones cognitivas y motrices implicadas. Por otro lado, se necesitaba analizar el grado de dificultad de cada subtarea, de modo que se pudiera valorar el ajuste de la ayuda proporcionada por el profesor. Se consideraron dos niveles de dificultad intrínseca asociados a la diferente complejidad de las subtareas. Se categorizó como grado de dificultad bajo (GDB), aquella subtarea requería activar conocimientos previos relativamente simples o accesibles para la mayoría de los estudiantes de ese nivel educativo. Se consideró como grado de dificultad mediaalta (GDMA) cuando la comprensión básica de la subtarea a realizar requería activar conocimientos previos supuestamente complejos o con mayores riesgos para la salud del paciente. La Tabla 4 contiene ejemplos de estas categorías.

El grado de dificultad de cada subtarea fue analizado y acordado por la investigadora, junto con los profesores participantes, resultando con mayor grado de dificultad los coincidentes con los puntos críticos de cada procedimiento.

TABLA 4

Sistema de categorías según el nivel de dificultad

\begin{tabular}{|c|c|c|}
\hline Procedimiento & $\begin{array}{c}\text { DIFICULTAD } \\
\text { SUBTAREA }\end{array}$ & TRANSCRIPCIÓN \\
\hline \multirow[t]{2}{*}{$\begin{array}{l}\text { Procedimiento } \\
\text { de la punción } \\
\text { venosa }\end{array}$} & GDB & $\begin{array}{l}\text { Profesora: [...] Ahora como ha pasado un tiempo desde } \\
\text { que le palpé al paciente hasta ahora, si yo lo quiero hacer } \\
\text { bien, la única manera que hay es que como voy a necesitar } \\
\text { palpar otra vez, lo que hago es que me desinfecto con otra } \\
\text { de estas gasas que había dejado aquí con el medic... con } \\
\text { el antiséptico, me desinfecto estos dos dedos y los puedo } \\
\text { utilizar para palpar. Cogemos la aguja con el bisel hacia } \\
\text { arriba ¿veis? Y palpamos. [...] }\end{array}$ \\
\hline & GDMA & $\begin{array}{l}\text { Profesora: [...] Y... pinchamos en un ángulo de } 45^{\circ} \text {. } \\
\text { Hombre, siempre en el muñeco, tanto la piel como las } \\
\text { venas que lleva por dentro, el sistema que lleva de venas } \\
\text { es distinto, entonces en el muñeco siempre se pincha con } \\
\text { un poco más de ángulo. Hay que tenerlo en cuenta. [...] }\end{array}$ \\
\hline
\end{tabular}


MARÍA JOSEFA BENAVENTE SANGUINO, MANUEL LUCERO FUSTES Y SIXTO CUBO DELGADO

ANÁLISIS DE LA INTERACCIÓN VERBAL ENTRE PROFESORES Y ESTUDIANTES DE ENFERMERÍA EN CONTEXTOS DE AULA Y HOSPITAL. UN ESTUDIO DESCRIPTIVO

\begin{tabular}{|c|c|c|}
\hline PROCEDIMIENTO & $\begin{array}{c}\text { DIFICULTAD } \\
\text { SUBTAREA }\end{array}$ & TRANSCRIPCIÓN \\
\hline & GDB & $\begin{array}{l}\text { Profesor: [...] Bien. Se recomienda que al menos que esté } \\
\text { tranquilo ¿no?, que no esté recién comido y que al menos } \\
\text { lleve cinco minutos de descanso, que no venga ligero a } \\
\text { tomarse la P.A. y de pronto se le toma ¿no? Porque todos } \\
\text { esos factores varían luego los valores de la P.A. [...] }\end{array}$ \\
\hline $\begin{array}{l}\text { Procedimiento } \\
\text { de toma de la } \\
\text { presión arterial }\end{array}$ & GDMA & $\begin{array}{l}\text { Profesor: [...] se infla el manguito a } 20 \text { o } 30 \mathrm{~mm} \text { por encima } \\
\text { de donde hemos dejado de detectar el pulso, empezamos } \\
\text { a liberar aire. ¿Con qué velocidad empezamos a liberar } \\
\text { aire? La velocidad recomendada es entre } 2 \text { y } 3 \mathrm{~mm} \text { de } \\
\text { mercurio ¿uhm?... 2-3 mm de, de mercurio por segundo y, } \\
\text { y estamos atentos a escuchar un primer latido. El primer } \\
\text { latido pues, en la situación en que se encuentre la aguja, } \\
\text { esa es la sistólica y continuamos escuchando latidos. Hay } \\
\text { un momento que desaparece. En el momento ese de } \\
\text { desaparecer, esa es la presión diastólica ¿de acuerdo? }\end{array}$ \\
\hline
\end{tabular}

GDB: grado de dificultad bajo; GDMA: grado de dificultad medio-alto.

Dimensión 2. Categorización de las subtareas según el grado de participación

Por otro lado, se analizó el grado de responsabilidad o autonomía verbal que el estudiante asume en la elaboración de una determinada subtarea. Se trata de categorizar diferentes niveles de participación verbal en el contenido que se hace público, tanto del profesorado como de los estudiantes. Esto permite inferir diferentes niveles de traspaso del control o apropiación de los procesos implicados en la ejecución de las operaciones, es decir, la implicación del estudiante en la autorregulación de los procedimientos de Toma de la Presión Arterial y Punción Venosa. Categorizar una intervención verbal con un nivel de participación bajo (GPB) implica que el estudiante no tiene participación verbal alguna, limitándose a escuchar al profesor, tomar notas o responder a preguntas cerradas (por lo tanto, es el profesor quien elabora la información que se hace pública en la sala de demostración o en la unidad de enfermería). Una subtarea categorizada con un grado de participación media (GPM) requiere por parte del estudiante responder a preguntas más abiertas inducidas por el profesor sobre cada subtarea. Finalmente, en un grado de participación alto (GPA), el estudiante es el responsable de la verbalización de los pasos de la subtarea, lo que supone, por otro lado, un evidente indicio de autorregulación. En la Tabla 5 se describen y ejemplifican las tres categorías que se utilizaron para analizar esta dimensión. 
MARÍA JOSEFA BENAVENTE SANGUINO, MANUEL LUCERO FUSTES Y SIXTO CUBO DELGADO ANÁLISIS DE LA INTERACCIÓN VERBAL ENTRE PROFESORES Y ESTUDIANTES DE ENFERMERÍA EN CONTEXTOS DE AULA Y HOSPITAL. UN ESTUDIO DESCRIPTIVO

TABLA 5

Sistema de categoría según el nivel de participación

\begin{tabular}{|c|l|}
\hline PARTICIPACIÓN & \multicolumn{1}{|c|}{ EJEMPLOS (TAREA DE PUNCIÓN VENOSA) } \\
\hline Baja & $\begin{array}{l}\text { PE: Desinfecto un poco, coloco el compresor... y ahora me coloco } \\
\text { los guantes. Me voy colocando los guantes para que mientras tanto, el } \\
\text { medicamento, el desinfectante se vaya secando. }\end{array}$ \\
\hline Media & $\begin{array}{l}\text { EE: Si es que me va a quedar grande... si es que yo no sé... quizá... [...] } \\
\text { Este es el 7-8 [...] Vale. }\end{array}$ \\
\hline Alta & $\begin{array}{l}\text { EE: }[\ldots] \text { Compresor... el bote para la técnica que fuéramos a realizar, } \\
\text { esparadrapo, gasas, palomillas... El material según el manual de procedimientos } \\
\text { hay que prepararlo doble por si algo... [...] falla o lo que sea, que no haya que } \\
\text { volver... [...] Y luego la campana y el alcohol que va a ser compartido... }\end{array}$ \\
\hline
\end{tabular}

PE: profesor de enfermería; EE: estudiante de enfermería.

\subsubsection{Medidas}

Como hemos descrito anteriormente, pretendemos conocer y comparar qué diferencias existen en las explicaciones facilitadas por el profesorado de los distintos contextos en torno a la realización de los dos procedimientos seleccionados, cómo se enfatiza en los puntos críticos establecidos como grados de dificultad del procedimiento y qué participación asume el estudiante en todo el proceso (quién).

En cuanto al qué y al cómo, ciñéndonos estrictamente a las transcripciones obtenidas, pueden señalarse al menos tres indicadores que aportan información acerca de las diferencias de las exposiciones efectuadas en los diferentes entornos: a) la cantidad de explicaciones e intervenciones verbales centradas en la realización de las 20 subtareas de cada procedimiento; b) el número de explicaciones e intervenciones correspondientes a las subtareas que se llevan a cabo antes, durante y después de cada procedimiento, como indicadores de un proceso que va del pensamiento a la acción, centrado en el paciente y en su seguridad, no en una mera ejecución mecánica; c) la cantidad de explicaciones e intervenciones emitidas en torno a las subtareas con diferente grado de dificultad.

El otro aspecto a considerar, vinculado al aprendizaje del estudiante, tiene que ver con los procesos de traspaso del control que se ponen de manifiesto a través de la asunción progresiva de responsabilidades en la ejecución de las subtareas, así como la de conseguir un suficiente grado de autonomía al finalizar el proceso. Para ello se cuenta con un indicador principal: el número de intervenciones con GPB, GPM y GPA en el desarrollo de cada procedimiento por parte del estudiante. 
MARÍA JOSEFA BENAVENTE SANGUINO, MANUEL LUCERO FUSTES Y SIXTO CUBO DELGADO

ANÁLISIS DE LA INTERACCIÓN VERBAL ENTRE PROFESORES Y ESTUDIANTES DE ENFERMERÍA EN CONTEXTOS DE AULA Y HOSPITAL. UN ESTUDIO DESCRIPTIVO

\subsubsection{Fiabilidad}

Los investigadores examinaron independientemente una muestra de los registros audiovisuales y las correspondientes transcripciones, con objeto de estimar el grado de concordancia en las diferentes categorizaciones. Las discrepancias se discutieron y se resolvieron por consenso entre los investigadores. Tras un proceso de entrenamiento, en el que los investigadores afinaron el sistema de categorías y el grado de apertura de las mismas, se obtuvieron medidas de fiabilidad bastante altas. Los índices de acuerdo interjueces (Kappa de Cohen) obtenidos en varias muestras de los registros con distintos procedimientos (Toma de la Presión Arterial y Punción Venosa) y subtareas fueron superiores a 0,9 ( $\mathrm{p}<0,0001)$.

\section{Resultados}

A continuación se presentan los resultados de las interacciones o verbalizaciones que se produjeron entre los estudiantes y los profesores de la Escuela o de la Unidad de Enfermería, durante el desarrollo de las sesiones de aprendizaje de los dos procedimientos seleccionados.

\subsection{Distribución general de las verbalizaciones por subtareas y procedimientos}

En las grabaciones efectuadas durante las sesiones sobre la presión arterial se registraron 184 intervenciones, mientras que en el procedimiento clínico de punción venosa se registraron 217 exposiciones verbales. La distribución de estas verbalizaciones, según la subtarea y contexto donde se produjeron, se ve reflejada en la Tabla 6.

Con respecto al procedimiento de medición de la presión arterial, la subtarea que presenta más frecuencia tanto en valores absolutos como porcentualmente es la 16: liberar la presión del manguito a una velocidad de 2-3 $\mathrm{mm}$ de $\mathrm{Hg} / \mathrm{s}$, para poder escuchar los latidos que marcan la presión arterial (33,8\% frente a un 19\% en la Unidad de Enfermería). Las subtareas de identificar al paciente o comentar con él las cifras de presión arterial no se han realizado en la Sala de Demostración. Puede tener explicación si se tiene en cuenta que no se trabaja con pacientes reales.

En la Unidad de Enfermería se aprecia la ausencia de verbalizaciones referidas a la localización del pulso braquial, vaciar el aire del manguito, insuflar el aire en el manguito sin quitar los dedos de la arteria y guardar el equipo y registrar los valores en la gráfica del paciente. Puede observarse que la subtarea 18, dejar al paciente en posición cómoda y con el brazo cubierto, es la que más se recoge en las interacciones entre la enfermera y la estudiante. Tanto en la Sala de Demostración como en la Unidad de Enfermería, no se recogen verbalizaciones acerca de las subtareas 3 y 4, referidas a la comprobación de las órdenes y el lavado de manos. Esta última es especialmente significativa e importante en el sistema sanitario por cuanto contribuye a garantizar la seguridad del paciente. Llama la atención, 
MARÍA JOSEFA BENAVENTE SANGUINO, MANUEL LUCERO FUSTES Y SIXTO CUBO DELGADO ANÁLISIS DE LA INTERACCIÓN VERBAL ENTRE PROFESORES Y ESTUDIANTES DE ENFERMERÍA EN CONTEXTOS DE AULA Y HOSPITAL. UN ESTUDIO DESCRIPTIVO

por otro lado, que en ninguno de los dos contextos se verbalizan todas las subtareas implicadas en el desarrollo del procedimiento.

TABLA 6

Número total de interacciones verbales realizadas en los procedimientos clínicos. Las subtareas decisivas y más complicadas aparecen señaladas en las tablas con un asterisco (*)

\begin{tabular}{|c|c|c|c|c|c|}
\hline \multirow{2}{*}{ SUBTAREA } & \multicolumn{2}{|c|}{ PRESIÓN ARTERIAL } & \multirow{2}{*}{ SUBTAREA } & \multicolumn{2}{c|}{ PUNCIÓN VENOSA } \\
\cline { 6 - 7 } & Escuela & Hospital & & Escuela & Hospital \\
\hline 1 & 1 & 1 & 1 & 1 & 0 \\
\hline 2 & 6 & 1 & 2 & 31 & 10 \\
\hline 3 & 0 & 0 & 3 & 1 & 0 \\
\hline 4 & 0 & 0 & 4 & 2 & 0 \\
\hline 5 & 0 & 4 & 5 & 3 & 2 \\
\hline 6 & 2 & 1 & 6 & 0 & 4 \\
\hline 7 & 1 & 2 & 7 & 4 & 7 \\
\hline 8 & 3 & 1 & $* 8$ & 25 & 13 \\
\hline 9 & 4 & 3 & 9 & 7 & 2 \\
\hline$* 10$ & 18 & 0 & 10 & 2 & 0 \\
\hline 11 & 1 & 0 & 11 & 3 & 0 \\
\hline 12 & 8 & 5 & 12 & 9 & 0 \\
\hline 13 & 6 & 1 & 13 & 15 & 5 \\
\hline 14 & 18 & 3 & $* 14$ & 3 & 3 \\
\hline 15 & 19 & 0 & 15 & 2 & 6 \\
\hline$* 16$ & 48 & 5 & 16 & 2 & 23 \\
\hline 17 & 4 & 4 & 17 & 5 & 1 \\
\hline 18 & 1 & 8 & 18 & 2 & 1 \\
\hline 19 & 0 & 3 & 19 & 1 & 11 \\
\hline 20 & 2 & 0 & 20 & 6 & 5 \\
\hline Total & 142 & 42 & Total & 124 & 93 \\
\hline & & & & & \\
\hline
\end{tabular}

Por otro lado, en cuanto al procedimiento de la punción venosa, se observa que la subtarea verbalizada con mayor frecuencia es la 2, seleccionar el material adecuado, contabilizándose este dato en la Sala de Demostración. En la Unidad de Enfermería, parece que lo que más preocupa en atención a las intervenciones cuantificadas es la subtarea 16, extraer la cantidad de sangre indicada para la prueba requerida y quitar el compresor antes de retirar la aguja. Otro dato que merece consideración es que en ambos lugares se contabilizan un gran número de intervenciones relacionadas con la subtarea 8, coincidente con uno de los puntos críticos del procedimiento: colocar el compresor para valorar las venas antes de 
MARÍA JOSEFA BENAVENTE SANGUINO, MANUEL LUCERO FUSTES Y SIXTO CUBO DELGADO

ANÁLISIS DE LA INTERACCIÓN VERBAL ENTRE PROFESORES Y ESTUDIANTES DE ENFERMERÍA EN CONTEXTOS DE AULA Y HOSPITAL. UN ESTUDIO DESCRIPTIVO

realizar la técnica. Luego se retira. Tanto en la Escuela como en el Hospital, esta subtarea ocupa el segundo lugar en la frecuencia de intervenciones.

En cuanto al segundo punto crítico, insertar la aguja en la vena seleccionada, con un ángulo de 30 a 45을 en ambos lugares la frecuencia es la misma. Por lo abultado del número, hay que fijarse en la subtarea 19, interesarse por el estado del paciente y dejarle cómodamente instalado, que vuelve a hacernos considerar que el confort y bienestar del paciente es altamente valorado en las Unidades de Enfermería.

\subsection{Interacciones en torno a las subtareas del antes, durante y después}

En la Tabla 7 se recoge el número de intervenciones verbales localizadas en torno a las subtareas agrupadas en antes, durante y después de ambos procedimientos. Las subtareas establecidas para el inicio del procedimiento, el antes, son siete; diez corresponden al durante, y tres se desarrollan después de ejecutar el procedimiento, aunque forman parte de él. Hay que considerar, por tanto, que el 50\% de las subtareas del procedimiento corresponden a las que se ejecutan durante el desarrollo del mismo.

TABLA 7

Interacciones verbales realizadas antes, durante y después del procedimiento

\begin{tabular}{|c|c|c|c|c|c|}
\hline PROCEDIMIENTO & SUBTAREAS & ANTES & DURANTE & DESPUÉS & TOTAL \\
\hline \multirow{2}{*}{ Presión arterial } & Escuela & $10(7 \%)$ & $129(90,9 \%)$ & $3(2,1 \%)$ & 142 \\
\cline { 2 - 6 } & Hospital & $9(21,5 \%)$ & $22(52,3 \%)$ & $11(26,2 \%)$ & 42 \\
\hline \multirow{2}{*}{ Punción venosa } & Escuela & $42(33,9 \%)$ & $73(58,9 \%)$ & $9(7,2 \%)$ & 124 \\
\cline { 2 - 6 } & Hospital & $23(24,7 \%)$ & $53(57 \%)$ & $17(18,3 \%)$ & 93 \\
\hline
\end{tabular}

Tanto en la Sala de Demostración de la Escuela como en la Unidad de Enfermería, el mayor número de verbalizaciones corresponden a las subtareas llevadas a cabo durante el desarrollo del procedimiento, posiblemente por la causa aludida. No obstante, es igualmente significativo que en la Sala de Demostración casi se duplican las intervenciones referidas a la etapa de ejecución (durante) del procedimiento con relación a las que se llevan a cabo en la Unidad de Enfermería.

\subsection{Interacciones según el grado de dificultad de cada subtarea}

Conviene recordar que se establecieron dos grados de dificultad coincidentes con los puntos críticos de cada procedimiento. Como puede verse en la Tabla 8, en cuanto a las verbalizaciones relacionadas con los grados de dificultad establecidos, son más numerosas las de GDB en ambos contextos y procedimientos, especialmente en la Unidad de Enfermería. 
134 MARÍA JOSEFA BENAVENTE SANGUINO, MANUEL LUCERO FUSTES Y SIXTO CUBO DELGADO ANÁLISIS DE LA INTERACCIÓN VERBAL ENTRE PROFESORES Y ESTUDIANTES DE ENFERMERÍA EN CONTEXTOS DE AULA Y HOSPITAL. UN ESTUDIO DESCRIPTIVO

TABLA 8

Interacciones verbales realizadas en torno a las subtareas según el grado de dificultad

\begin{tabular}{|c|c|c|c|c|}
\hline PROCEDIMIENTO & SUBTAREAS & GDB & GDMA & TOTAL \\
\hline \multirow{2}{*}{ Presión arterial } & Escuela & $76(53,5 \%)$ & $66(46,5 \%)$ & 142 \\
\cline { 2 - 5 } & Hospital & $37(88 \%)$ & $5(12 \%)$ & 42 \\
\hline \multirow{2}{*}{ Punción venosa } & Escuela & $96(77,4 \%)$ & $28(22,6 \%)$ & 124 \\
\cline { 2 - 5 } & Hospital & $77(82,8 \%)$ & $16(17,2 \%)$ & 93 \\
\hline
\end{tabular}

GDB: grado de dificultad bajo; GDMA: grado de dificultad medio-alto.

\subsection{Verbalizaciones según el grado de participación del estudiante}

Recuérdese que el grado de participación trataba de categorizar diferentes niveles de participación verbal en el contenido expuesto por parte de los estudiantes. Se establecieron tres niveles (bajo, medio y alto), que informan de la implicación gradual del estudiante en las verbalizaciones. Oscilan entre el GDB, en el que solo interviene el profesor o la enfermera, al GDA, que cataloga las verbalizaciones exclusivas del estudiante en la ejecución de la subtarea. Con este último indicador analizado, se intenta averiguar si la participación del estudiante se incrementa progresivamente junto con el aumento de responsabilidades en la ejecución de las subtareas.

En los datos reflejados en la Tabla 9, se observa que tanto para el procedimiento de la presión arterial como en el de la punción venosa, el grado de participación predominante es bajo (GPB), tanto en el contexto de la escuela como del hospital $(71,8 \%$ en la escuela y $64,3 \%$ en el hospital para el procedimiento de presión arterial y $58,9 \%$ en la escuela y 55,9\% en el hospital para la punción venosa).

TABLA 9

Interacciones verbales relacionadas con el grado de participación

\begin{tabular}{|c|c|c|c|c|c|}
\hline PROCEDIMIENTO & SUBTAREAS & GPB & GPM & GPA & TOTAL \\
\hline \multirow{2}{*}{ Presión arterial } & Escuela & $102(71,8 \%)$ & $18(12,8 \%)$ & $22(15,5 \%)$ & 142 \\
\cline { 2 - 6 } & Hospital & $27(64,3 \%)$ & $7(16,7 \%)$ & $8(19 \%)$ & 42 \\
\hline \multirow{2}{*}{ Punción venosa } & Escuela & $73(58,9 \%)$ & $20(16,1 \%)$ & $31(25 \%)$ & 124 \\
\cline { 2 - 6 } & Hospital & $52(55,9 \%)$ & $8(8,6 \%)$ & $33(35,5 \%)$ & 93 \\
\hline
\end{tabular}

GPB: grado de participación bajo; GPM: grado de participación medio; GPA: grado de participación alto. 
MARÍA JOSEFA BENAVENTE SANGUINO, MANUEL LUCERO FUSTES Y SIXTO CUBO DELGADO

ANÁLISIS DE LA INTERACCIÓN VERBAL ENTRE PROFESORES Y ESTUDIANTES DE ENFERMERÍA EN CONTEXTOS DE AULA Y HOSPITAL. UN ESTUDIO DESCRIPTIVO

El Gráfico 1 refleja de forma más clara dicha participación.

GRÁFICO 1

Distribución de las interacciones verbales según el grado de participación

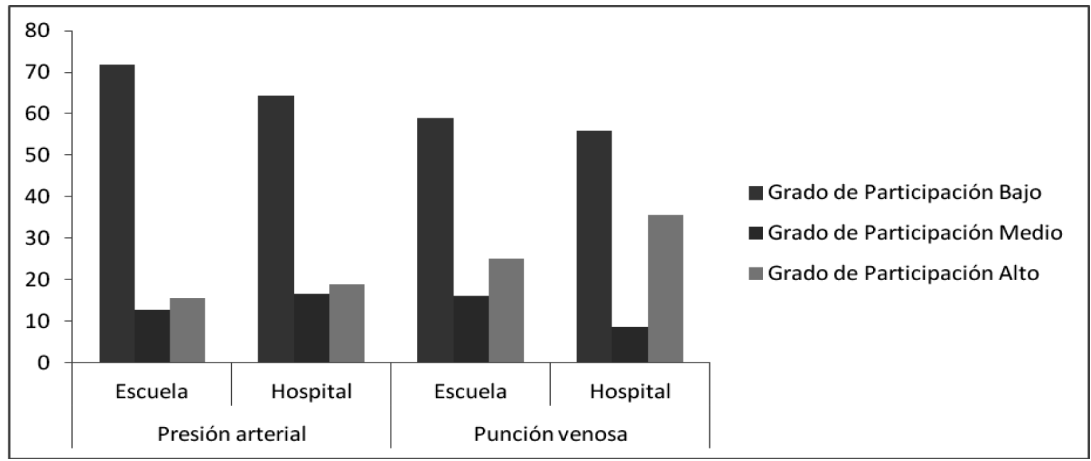

\section{DisCUSIÓN}

En este estudio pretendíamos caracterizar y describir el discurso de los profesores de Enfermería en torno al contenido de dos procedimientos (Toma de la Presión Arterial y Punción Venosa) en las Unidades de Enfermería de un hospital y en el aula universitaria. Para ello se han analizado los indicadores propuestos en el estudio, considerando uno a uno los dos casos planteados. Se han examinado todas las interacciones en torno a las subtareas de cada procedimiento; se ha indagado en las subtareas que conforman el antes, durante y después de cada práctica; se han comparado las participaciones con diferente grado de dificultad, y se ha intentado profundizar en el proceso de traspaso de control de los estudiantes. A continuación intentaremos interpretar los anteriores resultados en torno a los dos objetivos específicos que habíamos planteado.

Por un lado, el procedimiento de análisis de los registros verbales nos ha conducido a interpretar que las interacciones analizadas en este estudio en torno a las subtareas con grado de dificultad medio y alto se centran en los puntos críticos de ambos procedimientos clínicos. Estas verbalizaciones son más frecuentes en la Sala de Demostración que en las Unidades de Enfermería. Una explicación evidente de este hecho sería la importancia que el profesorado de esta Escuela de Enfermería otorga al aprendizaje de los puntos críticos establecidos para cada procedimiento, es decir, que durante el periodo de adiestramiento de los estudiantes en la Sala de Demostración, estas subtareas constituyen el foco principal de las verbalizaciones del profesorado.

Se advierte también que si consideramos los momentos establecidos en los procedimientos, es decir, el antes, el durante y el después, los aspectos previos correspondientes al antes son muy similares en ambos contextos; el desarrollo 
MARÍA JOSEFA BENAVENTE SANGUINO, MANUEL LUCERO FUSTES Y SIXTO CUBO DELGADO ANÁLISIS DE LA INTERACCIÓN VERBAL ENTRE PROFESORES Y ESTUDIANTES DE ENFERMERÍA EN CONTEXTOS DE AULA Y HOSPITAL. UN ESTUDIO DESCRIPTIVO

de la técnica (lo que acontece durante la ejecución del procedimiento) es mucho más importante en esta Escuela que en este Hospital, y los momentos finales (el después) de los procedimientos son, en los dos casos estudiados, mucho más significativos en las Unidades de Enfermería que en la Sala de Demostración, pudiendo concluirse que los aspectos técnicos priman en la Escuela y los referidos al confort y bienestar del paciente en el Hospital.

Por otro lado, en cuanto al apoyo a los procesos de traspaso de control, encontramos más diferencias entre las Unidades de Enfermería del Hospital y la Sala de Demostración de la Escuela. La descripción de las situaciones revela características comunes entre los dos procedimientos del estudio en cuanto al contenido y la estructura de la interacción, aunque también evidentes diferencias. Si bien, teóricamente, el estudiante, para conseguir una progresiva autonomía de traspaso de control, debería ir enfrentándose a subtareas cada vez más complejas al tiempo que disminuye el grado de ayuda que recibe por parte de los profesores, esto no ha sucedido así en los dos contextos en los que se han tomado las interacciones verbales. Los profesores de la Escuela de Enfermería desarrollaron una actividad de apoyo muy técnica y estructurada con los alumnos. Apenas se constataron episodios que mostraran una interacción más o menos simétrica o con un grado de participación alto de los estudiantes en el que éstos demandaran la ayuda específica que necesitaban. Al contrario de lo que se esperaba encontrar, en las Unidades de Enfermería el número de interacciones correspondientes a la subtareas con grado de participación medio y alto ha sido superior a la participación en la Sala de Demostración. Pudiera inferirse de los datos encontrados que las enfermeras que han participado en este estudio y que pertenecen al Hospital favorecen e incrementan el nivel de autonomía del estudiante. Por ello, podemos afirmar que de forma gradual se incrementa el nivel de responsabilidad y la autonomía de los alumnos al pasar de la Escuela a las Unidades de Enfermería que han sido referencia de este estudio.

En este contexto de la enseñanza y aprendizaje de la Enfermería, los errores que los alumnos cometen mientras están desarrollando los diferentes procedimientos pueden constituir referentes óptimos a partir de los cuales los profesores pueden efectivamente ajustar las ayudas que proporcionan a sus alumnos. Desde esta perspectiva, el procedimiento metodológico seguido en este estudio puede ayudar a clarificar los mecanismos interactivos mediante los cuales los profesores consiguen ajustar su ayuda verbal al proceso de construcción que llevan a cabo los alumnos analizados (Coll y Onrubia, 1997), independientemente del contexto en el que se producen (Escuela u Hospital).

Nuestros resultados, aunque exploratorios, son coincidentes con otros estudios que manifiestan la importancia de la relación entre la enseñanza de la teoría y la práctica, así como la ya constatada escisión entre las mismas. Existe una discrepancia entre las teorías explícitas (que se desarrollan a lo largo del proceso de formación) e implícitas (en la posterior práctica profesional o en el ámbito del aprendizaje práctico), que deben ser tenidas en cuenta por parte del profesorado en el proceso de aprendizaje de los estudiantes en las prácticas clínicas (Shön, 1992). 
MARÍA JOSEFA BENAVENTE SANGUINO, MANUEL LUCERO FUSTES Y SIXTO CUBO DELGADO

ANÁLISIS DE LA INTERACCIÓN VERBAL ENTRE PROFESORES Y ESTUDIANTES DE ENFERMERÍA EN CONTEXTOS DE AULA Y HOSPITAL. UN ESTUDIO DESCRIPTIVO

Antes de finalizar, no podemos dejar de hablar de las limitaciones de este estudio de caso múltiple. La más importante concierne al alcance de los resultados presentados en este trabajo. Por una parte, no hay que olvidar las limitaciones derivadas del hecho de que nuestros datos surgen de un estudio de caso en situación natural, lo que obliga a ser prudentes respecto a la generalización de los resultados. Por otra, y como ya hemos mencionado, para el análisis del discurso de los profesores de Enfermería utilizamos en ocasiones datos escasos y poco homogéneos (número total de contribuciones; número de pasos en el procedimiento con grado de dificultad bajo, medio o alto; número de intervenciones con diferentes grados de participación), lo que plantea dudas sobre la fiabilidad de las tendencias detectadas y su interpretación.

\section{REFERENCIAS BIBLIOGRÁFICAS}

Andrew, N. y Robb, Y. (2011). The duality of professional practice in nursing: Academics for the 21st century. Nurse Education Today, 31 (5), 429-433. Consultado el 12 de junio, 2012, de la base de datos MeDline. http://dx.doi.org/10.1016/j.nedt.2010.08.013

Ashworth, P. y Longmate, M. (1993). Theory and practice: beyond the dichotomy. Nurse Education Today, 13 (5), 321-327. Consultado el 9 de enero, 2012, de la base de datos MEDLINE. http://dx.doi.org/10.1016/0260-6917(93)90071-9

Becerra, T.; Montanero, M. y Lucero, M. (2012). Empleo normalizado con apoyo. Investigación de diferentes recursos de apoyo natural a trabajadores con discapacidad intelectual en tareas laborales que requieren autorregulación. Badajoz: OED.

Benner, P. (1987). Práctica progresiva en Enfermería. Barcelona: Grijalbo.

Cabero, J.; Llorente, M. C. y Román, P. (2004). Tools of Communication in Blended-learning. Pixel-Bit. Revista de Medios y Educación, 23, 27-41.

Carnwell, R.; Baker, S. A.; Bellis, M. y Murray, R. (2007). Managerial perceptions of mentor, lecturer practitioner and link tutor roles. Nurse Educational Today, 27 (8), 923-932. Consultado el 23 de abril, 2012, de la base de datos MEDLINE. http://dx.doi.org/10.1016/i. hedt.2007.01.005

Coll, C. (2010). La centralidad de la práctica y la dualidad conocimiento teórico/conocimiento práctico. Infancia y Aprendizaje, 33 (2), 141-159.

Coll, C.; Colomina, R.; Onrubia, J. y Rochera, M. J. (1995). Actividad conjunta y habla. En P. Fernández Berroca y M. ${ }^{a}$ A. Melero Zabal (Comps.). La interacción social en contextos educativos (pp. 193-326). Madrid: Siglo XXI.

Coll, C.; De Gispert, I. y Rochera, M. J. (2010). Tópicos y cadenas: una aproximación al análisis de la construcción conjunta de significados en foros de conversación en línea. Cultura y Educación, 2010, 22 (4), 439-454. http://dx.doi.org/10.1174/113564010793351885

Coll, C. y Onrubia, J. (1997). La Construcción de significados compartidos en el aula: actividad conjunta y dispositivos semióticos en el control y seguimiento mutuo entre profesor y alumnos. En C. Coll y D. Edwards (Eds.). Enseñanza, aprendizaje y discurso en el aula. Aproximaciones al estudio del discurso educacional (pp. 53-73). Madrid: Fundación Infancia y Aprendizaje.

Coll, C.; Onrubia, J. y Mauri, T. (2008). Ayudar a aprender en contextos educativos: el ejercicio de la influencia educativa y el análisis de la enseñanza. Revista de Educación, 346, 33-70. 
138 MARÍA JOSEFA BENAVENTE SANGUINO, MANUEL LUCERO FUSTES Y SIXTO CUBO DELGADO ANÁLISIS DE LA INTERACCIÓN VERBAL ENTRE PROFESORES Y ESTUDIANTES DE ENFERMERÍA EN CONTEXTOS DE AULA Y HOSPITAL. UN ESTUDIO DESCRIPTIVO

Comité Consultivo para la Formación de Enfermeros de la Comisión de las Comunidades Europeas (1990). Orientaciones sobre mayor acercamiento entre teoría y práctica en los programas de formación de enfermeros de cuidados generales. Bruselas.

Connant, L. (1992). Closing the Practice-theory Gap. En L. Nicoll (Ed.). Perspectives on Nursing Theory (pp. 463-468). Philadelphia: Lippincot.

Consejo General de Enfermería de España (1992). Estudio sobre la situación de enfermería de España. Madrid: CGEE.

Consejo Internacional de Enfermeras (2008). Reducción de la brecha y mejora de la relación entre la formación y la práctica: Marco de trabajo para análisis y generación de soluciones. Documento inédito. Ginebra: CIE.

Cubero, R. (2010). Sobre el conocimiento y la práctica educativa. Infancia y Aprendizaje, 33 (2), 161-165. http://dx.doi.org/10.1174/021037010791114607

Cubero, R.; Cubero, M.; Santamaría, A.; De la Mata Benítez, M. L.; Ignacio, M. a J. y Prados, M. (2008). La educación a través de su discurso. Prácticas educativas y construcción discursiva del conocimiento en el aula. Revista de Educación, 346, 71-104.

Cubero, R.; Santamaría, A.; De la Mata, M.; Prados, M. M. y Bascón, M. A. (2005). La construcción del conocimiento psicológico: un estudio de la interacción y el discurso en las aulas universitarias. La creación y mantenimiento de la intersubjetividad. En A. Franzé y otros (Comps.). Etnografía y Educación (pp. 15-32) (versión digital). Valencia: Germania, S. L.

Edwards, D. y Mercer, N. (1988). El conocimiento compartido en el aula. El desarrollo de la comprensión en el aula. Madrid: Paidós.

García Aretio, L. (2003). Comunidades de aprendizaje en entornos virtuales: La comunidad iberoamericana de la CUED. En M. Barajas (Coord.). La tecnología educativa en la educación superior (pp. 171-199). Madrid: McGraw-Hill.

García, R.; Rosales, J. y Sánchez, E. (2003). El asesoramiento psicopedagógico como construcción de significados compartidos: un estudio sobre su dificultad. Cultura E Educación, 15 (2), 129-148. http://dx.doi.org/10.1174/113564003322253794

Jokelainen, M.; Turunen, H.; Tossavainen, K.; Jamookeeah, D. y Coco, K. (2011). This systematic review described mentoring of nursing students in clinical placements. Journal of Clinical Nursing, 20 (19-20), 2854-2867. Consultado el 17 de julio, 2012, de la base de datos MEDLINE. http://dx.doi.org/10.1111/j.1365-2702.2010.03571.x

Lucero, M. y Montanero, M. (2008). La explicación multicausal en el aula de Historia. Tres experiencias de asesoramiento psicopedagógico. Infancia y Aprendizaje, 31 (1), 45-65. http://dx.doi.org/10.1174/021037008783487057

Mercer, N. (2001). Palabras y mentes. Cómo usamos el lenguaje para pensar juntos. Barcelona: Paidós.

Montanero, M. y García, G. (2005). ¿Qué hacen los profesores cuando los alumnos se equivocan? Un análisis de la interacción verbal en el aula de apoyo. Infancia y Aprendizaje, 28 (2), 141-157. http://dx.doi.org/10.1174/0210370053699311

Montanero, M. y Lucero, M. (2011). Causal discourse and the teaching of history. How do teachers explain historical causality? nstructional Science, 39, 2, 109-136. http://dx.doi. org/10.1007/s11251-009-9112-y

Myall, M.; Levett-Jones, T. y Lathlean, J. (2008). Mentorship in contemporary practice: the experiences of nursing students and practice mentors. Journal of Clinical Nursing, 17 (14), 1834-1842. Consultado el 27 de febrero de 2012, de la base de datos MEDLine. http://dx.doi.org/10.1111/j.1365-2702.2007.02233.x 
MARÍA JOSEFA BENAVENTE SANGUINO, MANUEL LUCERO FUSTES Y SIXTO CUBO DELGADO

ANÁLISIS DE LA INTERACCIÓN VERBAL ENTRE PROFESORES Y ESTUDIANTES DE ENFERMERÍA

EN CONTEXTOS DE AULA Y HOSPITAL. UN ESTUDIO DESCRIPTIVO

Onrubia, J.; Naranjo, M. y Segués, M. T. (2009). Debate y construcción de conocimiento en foros virtuales: la importancia de los motivos de los participantes en la actividad. Cultura y Educación, 21 (3), 275-289. http://dx.doi.org/10.1174/113564009789052325

Sánchez, E.; García, R.; Rosales, J.; De Sixte, R. y Castellano, N. (2008). Elementos para analizar la interacción entre estudiantes y profesores: ¿qué ocurre cuando se consideran diferentes dimensiones y diferentes unidades de análisis? Revista de Educación, 346, $105-136$

Sánchez, E. y Rosales, J. (2005). La práctica educativa. Una revisión a partir del estudio de la interacción profesor-alumnos en el aula. Cultura y Educación, 17 (2), 147-173. http:// dx.doi.org/10.1174/1135640054192865

Sharples, K.; Kelly, D. y Elcock, K. (2007). Supporting mentors in practice. Nursing Standard (Royal College of Nursing), 21 (39), 44-47. Consultado el 16 de mayo de 2012, de la base de datos MEDLINE. http://dx.doi.org/10.7748/ns2007.06.21.39.44.c4567

Shepherd, A. (2012). A quantitative discourse analysis of studen-initiated checks of understanding during teacher-fronted lesson. Linguistics and Education, 23, 145-159. http:/ dx.doi.org/10.1016/j.linged.2011.09.003

Shön, D. A. (1992). La formación de Profesionales reflexivos. Hacia un nuevo diseño de la enseñanza y el aprendizaje en las profesiones. Barcelona: Paidós. 